\title{
Race, Ethnicity and Subjective Well-Being: Exploring the Disparities in Life Satisfaction Among Whites, Latinx, and Asians
}

\author{
Tim Wadsworth · Philip M. Pendergast
}

\begin{abstract}
While much research has been done on the causes and correlates of subjective wellbeing over the last two decades, a relatively small number of studies have addressed disparities in subjective well-being between various racial and ethnic groups. Recently more research has addressed the differences between blacks and whites, and begun to unpack the causes for these differences. A smaller number of studies have started to look at differences between white and Latinx respondents. In the present work we add to this literature by examining differences in life satisfaction between white, Latinx and Asian respondents, as well as the persistence of these differences after controlling for a variety of social, economic and lifestyle variables. After assessing how much of the racial and ethnic disparity between these groups can be explained by such factors, we present additional preliminary analysis that begins to explore the role of culture in understanding the relationship between race, ethnicity and subjective well-being.
\end{abstract}

Keywords: well-being; happiness; life satisfaction; race; ethnicity; Latinx; Asian

\section{Introduction}

In recent years, social scientists have done a good job of documenting the demographic characteristics associated with subjective well-being (SWB); usually measured with questions about overall happiness or life satisfaction. From this work we know that married individuals report higher levels of well-being than singles (Haring-Hidore et al. 1985; Wadsworth 2016), and that younger and older respondents report being happier and more satisfied than the middle aged (Dolan 2008). We also know that levels of SWB vary across racial and ethnic groups. On average, blacks and Latinx report lower levels of happiness and life satisfaction than whites (Campbell et al. 1976; Clemente and Sauer 1976; Thomas and Hughes 1986; Hughes and Thomas 1998; Coverdill et al. 2011), but these trends have changed over time (Iceland and Ludwig-Dehm 2018). With some exceptions (Hughes and Thomas 1998), scholars have demonstrated that the difference in SWB between blacks and whites has decreased since the 1970s, primarily as a result of increasing SWB among the black population.

Much less work has examined differences between whites and other non-black minority groups and the mechanisms that drive racial and ethnic differences in SWB (Barger et al. 2009; Coverdill et al. 2011). In the current work we draw on the Behavioral Risk Factor Surveillance System (BRFSS) to both document existing racial and ethnic disparities in well-being between whites, Latinx and Asians, and to evaluate potential mechanisms by testing the resilience of 
disparities when controlling for a variety of well-established correlates of SWB-characteristics that also tend to vary by race and ethnicity. We also start to explore whether the remaining differences can be fully or partially explained by cultural factors.

\subsection{Why race and ethnicity (may) matter}

Potential explanations for the relationship between race, ethnicity and SWB steer us back to the foundational work and underlying debates in the study of SWB. One of the primary points of contention in this literature has been the relative importance of transient vs. more enduring characteristics. The latter are considered to be long-term, relatively stable aspects of one's personality and psychological architecture and are often viewed as the result of genetic predispositions. A recent meta-analysis found that genetics account for $32-36 \%$ of the variation in SWB (Bartels 2015). So, while much social science research ignores the role of these characteristics due to challenges in modeling these factors (requiring twin, genotyped, or genome-wide studies), there is often an unspoken assumption that individuals start with a certain "baseline" of SWB from which other more transient characteristics exert their influence. These other characteristics include contexts, social experiences and short or long-term circumstances. These characteristics are subject to change and exhibit large amounts of variation across individuals, groups, places and time. They include things like marital and employment status, income, health, etc. The majority of the research on SWB focuses on variation explainable by these types of characteristics.

There is significant evidence that many of these contexts, social experiences and circumstances vary systematically by race and ethnicity and have long been used to discuss issues of racial and ethnic stratification. Income, educational achievement and employment status have been central to the study of racial and ethnic inequality. Indicators of marital status and family formation are often used in conjunction with these measures to assess concentrated disadvantage (Kubrin et al. 2006; Wilson 1990), and racial and ethnic health disparities have been widely studied in medical sociology and public health (Williams and Sternthal 2010). These and other racially and ethnically clustered characteristics are also strongly related to SWB. Marital status has consistently been one of the best predictors of happiness and life satisfaction (Wadsworth 2016), and physical health (Dolan et al. 2008), absolute and relative income (Clark et al. 2008), employment status (Dolan et al. 2008) and education (Blanchflower and Oswald 2004) have all been identified as correlates of SWB. Given that these factors vary by race and ethnicity and are also strongly associated with well-being, one explanation for the observed relationships between race/ethnicity and SWB is that these factors represent mechanisms that explain racial and ethnic disparities. If this is the case, the influence of race and ethnicity should be attenuated, and potentially eliminated once we control for many of these characteristics.

The second possibility is that some more enduring characteristic(s) drive the relationship between race/ethnicity and SWB - that above and beyond more transient conditions, non-whites are more likely to report lower, or in some cases, higher, levels of SWB. While the research on genetic explanations for racial and ethnic differences on broad psychological measures has been far from convincing (Tov and Diener 2009), it may be useful to consider another type of enduring characteristic - culture. While culture has not been discussed much in the literature on racial and ethnic disparities in SWB, there may be important and resilient cultural differences that can help explain these disparities.

International comparisons have demonstrated significant differences in SWB even after adjusting for economic and other macro-structural forces (Diener and Suh 1999; Diener and Oishi 2000). These differences are often thought to be the result of cultural differences in emotional 
expression norms or different prioritizations and influences of certain life domains. For instance, Eid and Diener (2001) found that the percentage of respondents who viewed pleasant emotions such as joy, affection, pride and contentment as desirable and/or appropriate varied significantly across the U.S., Australia, China and Taiwan. While 83\% of Australians and Americans regarded all four of the emotions as desirable and appropriate, only $9 \%$ of the Chinese and $32 \%$ of the Taiwanese felt this way. Tov and Diener (2009) suggest that such differences in emotional expression norms may explain why East Asian samples often report lower levels of SWB compared to samples of European or North and South American respondents. For instance, they note that while Japan has a higher standard of living and greater purchasing power than most Latin American nations, the Japanese generally report lower levels of SWB than their Latin American counterparts (Diener and Suh 1999; Diener and Oishi 2000). Consistent with these findings, using one-week period of experience sampling, Scollon et al. (2004) found more frequent experiences of pleasant affect among European- and Latinx Americans compared to Asians and Asian Americans.

Additional research on happiness and life satisfaction in Latin America has demonstrated much higher levels of SWB than would be expected given economic factors as well as other variables representing access to resources and opportunities (Rojas and Vega 2017; Beytia 2016). While emotional expression norms may contribute to these differences, researchers have focused more on a different set of cultural expectations that place higher priority on family and other relationships and a lower priority on financial success and accumulation. In an article entitled, "The Singularity of Latin American Patterns of Happiness," Beytia (2016) used data from the World Values Survey to demonstrate that, on average, Latin American respondents gain more happiness from human relationships and are more successful in cultivating them than respondents from other regions of the world. Beytia writes,

"money, both in its objective expression (income) and subjective (financial satisfaction), has less association with happiness in Latin America than in the other regions studied.... In addition, those Latin American and U.S. who respect and love their parents unconditionally are happier, and in Latin America there is a greater proportion of people in this situation." (2016: 25-26)

While there is less empirical work on the topic, it is also possible that historical events can influence the collective conscience of large groups of racial or ethnic minorities. A growing body of work focused on cultural trauma and collective identity argues that the traumatic experiences of large groups of similarly identified individuals can create values, narratives and beliefs among entire groups, even those who did not directly experience the trauma and may not have even been alive when it occurred (Alexander et al. 2004). While difficult to measure it is likely that a history of oppression, prejudice and discrimination may influence the collective experience of various emotional states for African Americans, Latinx, Asians and other minority groups. However, recent work has also demonstrated higher levels of culturally embedded resilience and optimism among some minority groups during specific time periods and/or crises. Graham and Pinto (2019) report higher levels of optimism and lower levels of stress and pain among recent low income black and Latinx respondents compared to their white counterparts. They suggest that this helps explain their lower levels of "deaths of despair" over the last decade.

To date, much of the empirical research on culture and SWB has pointed to potential differences between Eastern and Western cultures, and contrasted Latin American culture with that of the United States and Europe, though the work on collective trauma and other cultural psycho-social adaptations extends the possibility of such differences to include additional ethnic and racial minorities. Whether these findings can shed light on potential differences in SWB 
between racial and ethnic groups within the U.S. is hard to know, especially given the potential for much more nuanced subcultural differences within the broader culture. It would be easier to rule out the influence of culturally based factors if racial and ethnic differences can be explained by individual economic, social and demographic characteristics than to provide empirical evidence that culture is playing an important role in shaping differences given the difficulty in operationalizing potentially relevant subcultural differences with nationally representative survey data. However, in the current work we begin to explore the role of culture by examining language usage as a measure of acculturation.

\subsection{What We Know about Race, Ethnicity and Subjective Well-Being in the United States}

Racial (and more recently, ethnic) differences in SWB have been documented in the literature since the mid-1970s. In a general review of research on the quality of life in the U.S., Campbell et al. (1976) and Bracy (1976) cited multiple studies that showed higher average happiness and life satisfaction scores among whites than blacks. Drawing on 1973 GSS data, Clemente and Sauer (1976) also identified significant differences between black and white life satisfaction scores, with whites scoring higher even after controlling for a variety of socioeconomic and demographic variables. Six years later, drawing on data originally collected by Andrews and Withey (1976), Herzog et al. reported very similar findings (Herzog et al. 1982). In 1986, Thomas and Hughes responded to William Julius Wilson's claims in The Declining Significance of Race (Wilson 1980) by suggesting that we needed to look at both objective and subjective measures of well-being to truly understand shifting patterns of racial inequality. After noting important changes in the legal and social status of blacks in the U.S., they continued to document consistent racial gaps on various subjective quality of life measures available in the GSS, including general life satisfaction, a trust in people scale, an anomie scale, a general happiness item, a marital happiness item, and a measure of physical health (Thomas and Hughes 1986). They reported that even after controlling for a variety of indicators of social class and other variables, "For all of the six dependent variables predicted mean scores show that whites experience better psychological well-being and quality of life than blacks do." When they revisited their analyses a decade later with more recent data, their overall findings remained the same (Hughes and Thomas 1998). Consistent with this work, Stevenson and Wolfers (2012) demonstrated that while the SWB gap between blacks and whites narrowed from the 1970s through the first decade of the $21^{\text {st }}$ century, significant differences still remained.

Using data from the BRFSS, Barger et al. (2009) were some of the first researchers to include both black and Latinx respondents in an analysis of subjective well-being. Their findings suggested that, like African Americans, Latinx respondents experience lower average levels of SWB than whites, but that most of these differences can be accounted for by an array of socioeconomic characteristics. In an attempt to revisit, extend, and improve on the work of Hughes and Thomas, Coverdill et al. (2011) again turned to the GSS to examine the relationship between race and ethnicity and happiness. In doing so they made two important contributions. Most importantly, more recent data allowed them to examine similar questions pertaining to the SWB of Latinx (as a whole and grouped by country of origin-Mexico vs. Non-Mexico). They documented a Latinx/white gap in happiness that is similar to the gap between blacks and whites, is robust when various controls are included in the models, and is not dependent on Latinx country of origin. Their second contribution was more methodological. While most of the previous work in the area had used linear regression analysis when modeling ordinal data (Hughes and Thomas 1998; Barger et al. 2009), Coverdill et al. used partial proportional odds models, which allow for differential effects across various levels of an ordinal scale (e.g. race or 
other variables may differentially influence the likelihood of being "unhappy" vs. "somewhat happy" or "very happy", compared to the likelihood of being "unhappy" or "somewhat happy" vs. "very happy"). This improved analytic approach led to very similar findings regarding SWB among blacks and whites - while gaps have diminished over time, there remain substantial and significant differences in overall happiness and other well-being measures between the two groups.

In more recent work, Iceland and Ludwig-Dehm (2019) address both changes in the blackwhite gap in happiness over the last four decades and offer an assessment of the driving factors behind the remaining gaps. They point to "the moderate narrowing of substantial racial disparities...such as life expectancy, residential segregation, and neighborhood conditions" (2017:16) as the primary explanation for the decrease in the happiness gap between whites and blacks and to the importance of marital status and income in explaining the remaining racial disparity in SWB.

In sum, five points stand out when reviewing the current state of the literature on race/ethnicity and SWB:

1. Happiness and life-satisfaction vary by race and ethnicity, with whites generally reporting higher levels than other racial and ethnic groups.

2. Research among black and Latinx respondents has demonstrated that even after controlling for a variety of socio-economic characteristics, these differences often still remain.

3. For blacks, much of the gap (with whites) appears to be associated with other well-studied disparities, specifically income and marital status.

4. We know much less about differences between other racial and ethnic groups.

5. There has been virtually no research regarding how more culturally-based factors may help explain racial and ethnic differences within the U.S.

The current research attempts to build on this literature in a number of ways. First, like Barger et al. (2009) we use data from the Behavioral Risk Factor Surveillance System, which provides a number of new possibilities. The substantially larger yearly BRFSS samples allow us to include Asians in our analysis, a group that has not experienced the same levels of socio-economic disadvantage as blacks and Latinx. The BRFSS includes a measure of life satisfaction, which is different than the happiness measure that has been utilized in more recent analyses from the GSS. As previous research has suggested the possibility of different effects of race on happiness vs. life satisfaction, it may be instructive to explore the latter. The BRFSS geo-coded data also allow us to consider the influence of state of residence (the GSS only allows the identification of which of the nine regions of the US the respondent lives in). As has been noted in previous research, we tend to observe higher levels of SWB in some states compared to others (Oswald and $\mathrm{Wu}$ 2010). As there is unequal distribution of race/ethnicity by state, controlling for where respondents live will allow us to control for the confounding influence of geographic location.

Second, in addition to evaluating whether there are differences in SWB by race and ethnicity, we conduct analyses to help explain what factors contribute to observable differences. While we appreciate Coverdill et al.'s assessment regarding the preference for time-series data in order to disentangle the potential causes of well-being (Coverdill et al. $2011 \mathrm{pg}$. 801), the BRFSS provides a wide array of covariates associated with both race/ethnicity and well-being. Introducing them as controls in a series of models can help us understand what factors are associated with larger or smaller racial and ethnic differences in life satisfaction. Relatedly, we move away from previous work that has used self-rated health as an indicator, rather than a contributor to SWB 
(Thomas and Hughes 1986; Hughes and Thomas 1998; Coverdill et al. 2011), as we suggest that it is one of the strongest predictors of happiness and life satisfaction and is strongly associated with race and ethnicity (Williams and Sternthal 2010). As such, its absence as a covariate may lead to misspecified models.

Lastly, and perhaps most importantly, the BRFSS allows us to begin to explore the influence of culture. By examining the influence of one aspect of culture, we are able to identify whether less personal, potentially more enduring characteristics help explain any racial and ethnic differences in life satisfaction.

\section{Data and methods}

As noted above, the present work draws on data from the 2010 Behavioral Risk Factor Surveillance System (BRFSS), the largest ongoing, nationally-representative, telephone-based survey in the United States (Centers for Disease Control and Prevention 2010). States conduct their own data collection using State-level, random-digit dialed disproportionate stratified probability samples of the adult population. Accordingly, response rates vary considerably by State, from a minimum of $39 \%$ in Oregon to a maximum of $69 \%$ in Nebraska, with data weighted for noncoverage and nonresponse. The BRFSS contains extensive data on self-reported risk factors and behaviors related to physical and mental health, and is widely used by public health departments and other state and local entities concerned about better understanding their communities. While we would ideally prefer more recent data, 2010 was the last year in which the BRFSS included a question about life satisfaction for the full national sample. Given that the primary racial and ethnic groups we are interested in, Asians and Latinx, are non-randomly distributed among states, later years of BRFSS data that only collect life satisfaction in a few states fail to capture a representative sample of the population of interest. Nonetheless, these data have several unique advantages.

First, the BRFSS distinguishes between several racial and ethnic groups not available in the GSS and other smaller surveys. Sample sizes for many of these groups are quite small, but the sample of Asians is sufficiently large to allow for their inclusion as another comparison group. Due to the small samples, we combine all of the other racial groups into an "Other Race" category that is excluded from the models. Finally, the BRFSS consists of yearly samples of between 300,000 and 500,000 respondents. This gives us information from 451,075 individuals in 2010, with which we can estimate very precise averages for the racial/ethnic groups included in our study. Therefore, if we can account for significant differences between groups in the BRFSS data, we can say with a high degree of confidence that our findings are generalizable to the greater U.S. population.

\subsection{Dependent Variable}

While there are several related measures of SWB in the literature, the BRFSS collects data on life satisfaction. Prior research suggests that life satisfaction represents an enduring assessment of the roles played by access to tangible and intangible resources for satisfaction with life in general, which is relatively invariant to day-to-day changes in current moods and situational contexts (Diener and Ryan 2009). In contrast, concepts such as happiness are more situationally determined by moods like joy, delight, anger, and fear, while also being influenced by more global assessments of life satisfaction and the hedonistic evaluations of experiencing one's life as "good" or "bad" (Kahneman, 1999), virtuous or fulfilling (Diener and Ryan 2009).

The BRFSS asks respondents: 
In general, how satisfied are you with your life? (Response categories include: very satisfied, satisfied, dissatisfied, very dissatisfied, don't know/not sure, refused)

Most $(98.7 \%)$ provide a valid answer to the question. Amongst those who respond, many report being either "very satisfied" (46.2\%) or "satisfied" (48.4\%), with fewer reporting dissatisfaction $(4.3 \%)$ or being "very dissatisfied" $(1.1 \%)$ with their lives (descriptive statistics for Life Satisfaction and all other variables are listed in Table 1.) An initial examination of the distribution of life satisfaction for whites, Asians, and Latinx led us to combine these final two responses into a single "dissatisfied" category for the sake of parsimony and to ensure adequate sample sizes in all response categories across race/ethnicity.

Table 1. Descriptive statistics, BRFSS 2010

\begin{tabular}{|c|c|c|c|c|}
\hline & Total sample & Non-Hispanic White & Latinx & Asian \\
\hline & Mean & Mean & Mean & Mean \\
\hline \multicolumn{5}{|l|}{ Life Satisfaction } \\
\hline Dissatisfied & 0.05 & 0.05 & 0.05 & 0.03 \\
\hline Satisfied & 0.48 & 0.46 & 0.54 & 0.56 \\
\hline Very Satisfied & 0.47 & 0.49 & 0.42 & 0.41 \\
\hline \multicolumn{5}{|l|}{ Race/Ethnicity } \\
\hline White & 0.80 & 1.00 & - & - \\
\hline Asian & 0.04 & - & - & 1.00 \\
\hline Latinx & 0.16 & - & 1.00 & - \\
\hline \multicolumn{5}{|l|}{ Demographics } \\
\hline Age & 47.31 & 48.87 & 41.00 & 40.94 \\
\hline$(S D)$ & $(16.69)$ & $(16.73)$ & (15.03) & $(14.78)$ \\
\hline Female & 0.50 & 0.51 & 0.50 & 0.43 \\
\hline \multicolumn{5}{|l|}{ Income } \\
\hline$\$ 0-\$ 14,999$ & 0.09 & 0.07 & 0.23 & 0.07 \\
\hline$\$ 15,000-\$ 24,999$ & 0.15 & 0.13 & 0.27 & 0.09 \\
\hline$\$ 25,000-\$ 34,999$ & 0.10 & 0.10 & 0.13 & 0.06 \\
\hline$\$ 35,000-\$ 49,999$ & 0.14 & 0.14 & 0.12 & 0.12 \\
\hline$\$ 50,000+$ & 0.52 & 0.57 & 0.25 & 0.65 \\
\hline \multicolumn{5}{|l|}{ Education } \\
\hline$<\mathrm{HS}$ & 0.09 & 0.05 & 0.29 & 0.03 \\
\hline HS Graduate & 0.26 & 0.26 & 0.29 & 0.13 \\
\hline Some College & 0.26 & 0.27 & 0.22 & 0.17 \\
\hline College Graduate & 0.39 & 0.41 & 0.21 & 0.67 \\
\hline \multicolumn{5}{|l|}{ Employment } \\
\hline Employed & 0.61 & 0.61 & 0.59 & 0.67 \\
\hline Unemployed & 0.07 & 0.07 & 0.11 & 0.09 \\
\hline Student & 0.04 & 0.03 & 0.05 & 0.10 \\
\hline Retired & 0.08 & 0.07 & 0.13 & 0.06 \\
\hline Homemaker & 0.16 & 0.18 & 0.07 & 0.07 \\
\hline Can't Work & 0.05 & 0.05 & 0.05 & 0.01 \\
\hline
\end{tabular}




\begin{tabular}{lcccc} 
Marital Status & & & & \\
Married & 0.66 & 0.68 & 0.57 & 0.70 \\
Divorced & 0.08 & 0.09 & 0.07 & 0.03 \\
Widow & 0.05 & 0.06 & 0.03 & 0.02 \\
Separated & 0.02 & 0.01 & 0.04 & 0.01 \\
Never Married & 0.18 & 0.16 & 0.28 & 0.24 \\
Self-Rated Health & & & 0.24 \\
Health "Fair/Poor" & 0.15 & 0.13 & 0.76 & 0.09 \\
Health "Good/Excellent" & 0.85 & 0.87 & & 0.56 \\
Language of Interview & & & 0.97 \\
English Language & 0.93 & 1.00 & 0.44 & 0.00 \\
Spanish Language & 0.07 & 0.00 & 0.00 & 0.03 \\
$\quad$ Other Language & 0.00 & 0.00 & 25299 & 6288 \\
\hline N & 321417 & 289830 & & 0.96 \\
\hline
\end{tabular}

Note. Analytic sample. Descriptive statistics calculated with sampling weights.

\subsection{Independent Variables}

Our focal independent variable is race/ethnicity. Since our primary motivation is in examining and explaining previously underexplored racial/ethnic disparities in life satisfaction, we retain only non-Hispanic whites, Asians, and Latinx (those reporting Hispanic ethnicity, of any race) in the sample.

The BRFSS also measures an array of sociodemographic and economic characteristics that likely account for life satisfaction disparities between racial/ethnic groups. To capture differences in sample characteristics that may explain these disparities, we construct covariates for age (we also include an age-squared term to better capture the functional form of SWB across the life course, which research has found is curvilinear; Ferrer-i-Carbonell and Frijters 2004), gender (male $=0$, female $=1)$, income $(\$ 0-\$ 14,999=0, \quad \$ 15,000-\$ 24,999=1, \quad \$ 25,000-\$ 34,999=2, \quad \$ 35,000-$ $\$ 49,999=3, \$ 50,000+=4$ ), education (less than a high school diploma $=0$, high school graduate $=1$, some college $=2$, college graduate $=3$ ), employment (a series of binary indicators comparing being unemployed, a student, retired, unable to work, or a homemaker to being employed), marital status (binary indicators comparing being divorced, widowed, separated, or never married to being married), and self-rated health $(0=$ "poor" or "fair", $1=$ "good" or "excellent"). Of these, income is missing the most data (14.3\%). There is intermittent missing data across other measures as well, and after listwise deletion of missing data on all study measures and restricting the sample to non-Hispanic whites, Asians, and Latinx, the final analytic sample is 321,417 (71.3\% of the base sample). The BRFSS also includes complete data for state identifiers (categorical, containing FIPS codes) and the language spoken during the interview (binary measures for Spanish speakers and non-English, non-Spanish speakers compared to those interviewed in English), which we include in some models. We discuss the use of the language measures in more detail below.

\subsection{Analysis Strategy}

Life satisfaction and other similar SWB concepts tend to be measured categorically in survey data. There exist several strategies for modeling these types of outcomes, each with their own benefits and limitations depending on the aims of the study. We began by fitting an extension of the general linear model, conventional ordered logistic regression models (PLUMs: Polytomous 
Universal Models), to examine racial/ethnic differences in life satisfaction. However, an important assumption of these models is that independent variables display proportional odds across all levels of the dependent variable (i.e. the influence of an independent variable on the odds of being "dissatisfied" vs. "satisfied" or "very satisfied" would be the same as its influence on the odds of being "dissatisfied" or "satisfied" vs. "very satisfied"). An investigation of model residuals, Wald tests (Brant 1990), and crosstabs suggested that the distributions of life satisfaction were not constant across racial and ethnic groups, as expected; in other words, the assumption of proportional odds was consistently violated by the focal independent variables, regardless of what other controls were added to the models.

While multinomial logistic regression may be an attractive alternative in that it allows each variable in the model to exert different influences on every category of the outcome, this option is not very parsimonious since many sociodemographic, health, and lifestyle variables do exert similar influences across all levels of the dependent variables. To remedy this issue while also allowing for more in-depth analyses of how SWB varies across racial categories, we instead estimate ordered logit models with partial proportional odds using the "gologit2" package (Williams 2016) for STATA 14 (Statacorp 2015). This approach allows us to relax the assumption of proportional odds only for those variables that violate it while retaining the more parsimonious restriction for those that do not. Thus, independent variables not violating the proportional odds assumption produce one estimate of the odds associated with an individual belonging to any higher category of life satisfaction. For measures that violate this assumption, the models estimate $k-1$ coefficients where $k$ represents the number of categories of the dependent variable.

This strategy yields a 3-category outcome measure, with the first set of coefficients for independent variables (section A of Table 2) representing the odds of a respondent with a given characteristic reporting that they are dissatisfied versus being either satisfied or very satisfied. As such, this indicates the odds of indicating the lowest level of life satisfaction. The second set of coefficients (section B of Table 2) represents the odds of a respondent being very satisfied rather than satisfied or dissatisfied-thus indicating the highest level of SWB. While these models typically report this second contrast in terms of the odds of belonging to the two lowest groups relative to the odds of being in the highest group, we chose to invert this contrast so that interpretation would be more intuitive (i.e. higher odds = more life satisfaction; lower odd $s=$ less life satisfaction). To further ease interpretation, we report odds ratios for all models. Finally, we also account for the sampling frame of the BRFSS with the STATA "svy" commands to adjust for the survey's sampling weights nested within the appropriate strata and primary sampling units.

\section{Findings}

Sections A and B of Table 2 present the findings from six models from the BRFSS data. Section A presents odds ratios indicating the relative likelihood (with white respondents being the reference group) of being "Dissatisfied" (rather than "Satisfied" or "Very Satisfied"), while Section B indicates the relative likelihood of being "Very Satisfied" (rather than "Dissatisfied" or "Satisfied"). In Section A of Model 1, which only includes measures of race, ethnicity, age and gender, we can see that race and ethnicity are sometimes but not always associated with life satisfaction. Latinx respondents demonstrate about the same likelihood of dissatisfaction with life as white respondents and Asians are much less likely to be dissatisfied with their lives $(\mathrm{OR}=.56)$ than whites. When considering one end of the SWB spectrum, dissatisfaction with life, there appears to be no effect of being Latinx and a reduction of risk for Asians before controlling for other covariates. However, when we turn to the likelihood of being very satisfied (Section B 
of Model 1) with life, a different picture emerges. In our baseline model, Latinx respondents have $22 \%(\mathrm{OR}=.78)$ and Asians have $23 \%(\mathrm{OR}=.77)$ lower odds of reporting being very satisfied.

Models 2-6 in Sections A and B demonstrate the relationships between typical correlates of SWB and the likelihood of a respondent reporting that they are "dissatisfied" (Section A) or "very satisfied" (Section B). In each successive model we include additional covariates that were discussed above. Similar to findings in previous research, we observe that life satisfaction is associated with state of residence in the 2010 BRFSS. While we do not list state coefficients due to space restrictions, we note that model 6 includes fixed effects for state of residence.

Table 2. Ordinal logistic regression models predicting life satisfaction by respondent characteristics, BRFSS 2010

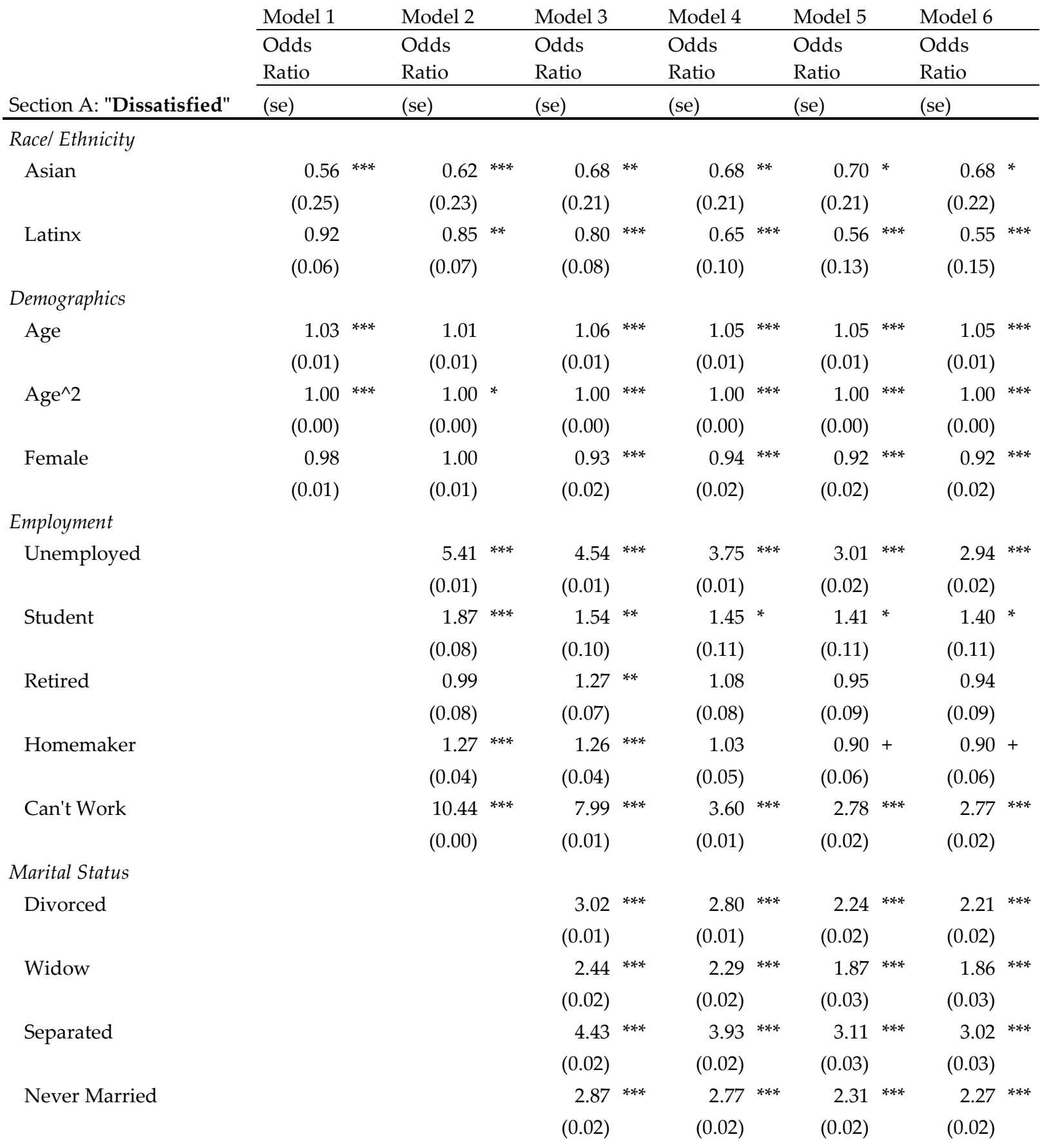




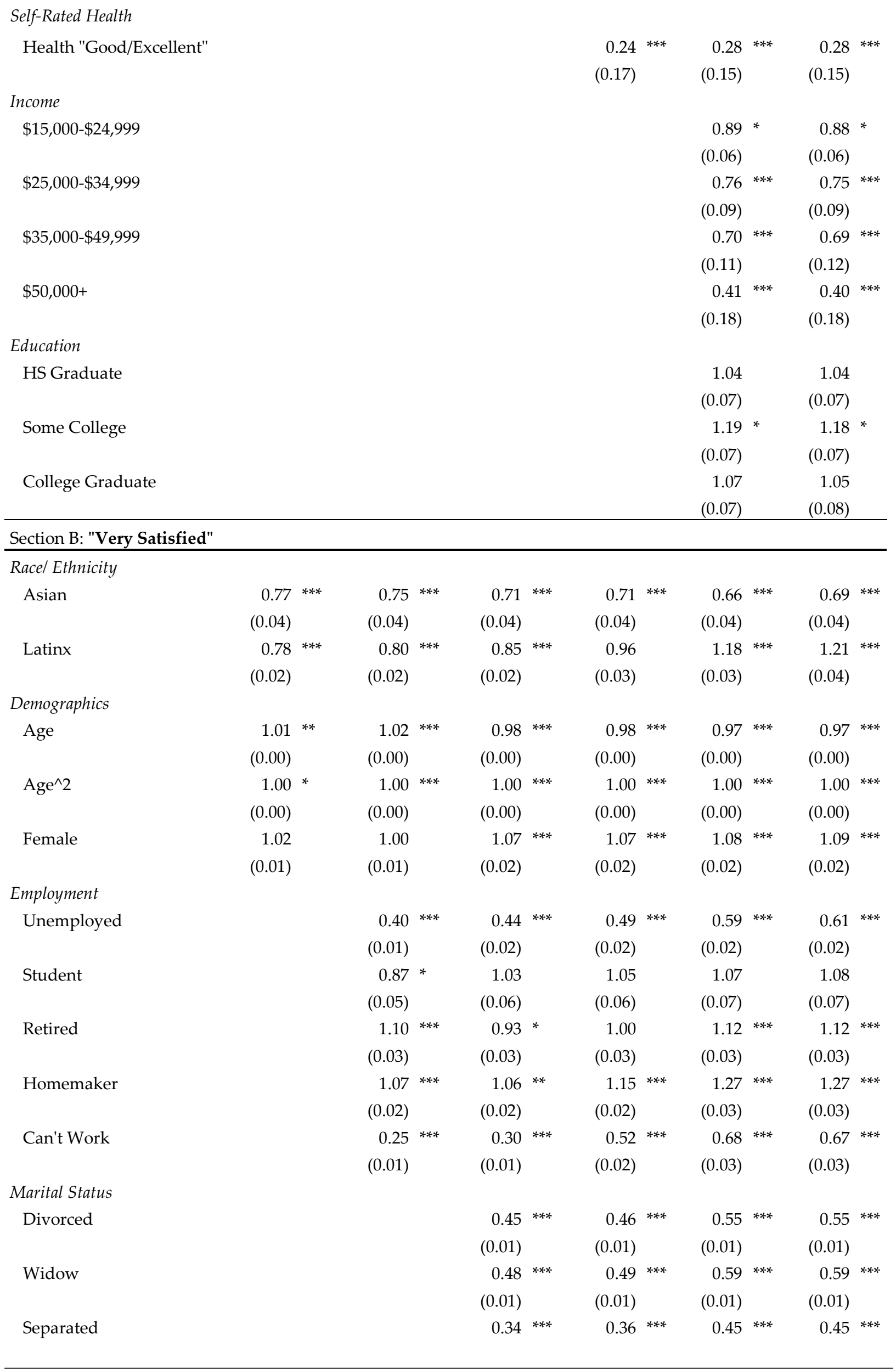




\begin{tabular}{|c|c|c|c|c|c|c|c|}
\hline & & & $(0.03)$ & $(0.03)$ & $(0.04)$ & $(0.04)$ & \\
\hline Never Married & & & $\begin{array}{l}0.48^{\text {*** }} \\
(0.01)\end{array}$ & $\begin{array}{l}0.49^{* * *} \\
(0.01)\end{array}$ & $\begin{array}{l}0.55^{* * *} \\
(0.01)\end{array}$ & $\begin{array}{r}0.56 \\
(0.02)\end{array}$ & $* * *$ \\
\hline \multicolumn{8}{|l|}{ Self-Rated Health } \\
\hline Health "Good/Excellent" & & & & $\begin{array}{l}2.75^{* * *} \\
(0.07)\end{array}$ & $\begin{array}{l}2.39 \text { *** } \\
(0.06)\end{array}$ & $\begin{array}{r}2.42 \\
(0.06)\end{array}$ & $* * *$ \\
\hline \multicolumn{8}{|l|}{ Income } \\
\hline$\$ 15,000-\$ 24,999$ & & & & & $\begin{array}{r}1.03 \\
(0.04)\end{array}$ & $\begin{array}{r}1.02 \\
(0.04)\end{array}$ & \\
\hline$\$ 25,000-\$ 34,999$ & & & & & $\begin{array}{r}1.06 \\
(0.04)\end{array}$ & $\begin{array}{r}1.06 \\
(0.04)\end{array}$ & \\
\hline$\$ 35,000-\$ 49,999$ & & & & & $\begin{array}{l}1.25^{* * *} \\
(0.05)\end{array}$ & $\begin{array}{r}1.26 \\
(0.05)\end{array}$ & $* * *$ \\
\hline$\$ 50,000+$ & & & & & $\begin{array}{l}1.81^{* * *} \\
(0.07)\end{array}$ & $\begin{array}{r}1.84 \\
(0.07)\end{array}$ & $* * *$ \\
\hline \multicolumn{8}{|l|}{ Education } \\
\hline HS Graduate & & & & & $\begin{array}{r}1.01 \\
(0.04)\end{array}$ & $\begin{array}{r}1.01 \\
(0.04)\end{array}$ & \\
\hline Some College & & & & & $\begin{array}{r}1.02 \\
(0.04)\end{array}$ & $\begin{array}{r}1.01 \\
(0.04)\end{array}$ & \\
\hline College Graduate & & & & & $\begin{array}{r}1.29 \\
(0.05)\end{array}$ & $\begin{array}{r}1.29 \\
(0.05) \\
\end{array}$ & $* * *$ \\
\hline Cut 1 Intercept & 0.03 & 0.03 & 0.01 & 0.03 & 0.04 & 0.02 & \\
\hline Cut 2 Intercept & 0.72 & 0.71 & 1.98 & 0.69 & 0.59 & 0.50 & \\
\hline $\mathrm{N}$ & 321417 & 321417 & 321417 & 321417 & 321417 & 321417 & \\
\hline $\begin{array}{l}\text { Note. Models adjust for san } \\
{ }^{*} \quad \mathrm{p}<0.05 \\
{ }_{* *} \quad \mathrm{p}<0.01 \\
* * * \quad \mathrm{p}<0.001\end{array}$ & $\overline{\text { ng weig }}$ & del 6 in & tate fixed e & & & & \\
\hline
\end{tabular}

As our primary interest in these covariates is whether they can help explain some or all of the racial and ethnic differences in life satisfaction that exist in the baseline model, we do not review the findings from all of the models in detail. Instead, we focus on how the relationship between race and ethnicity and life satisfaction changes after we control for potentially important social and economic characteristics. While it may be of interest to note the individual changes from one model to the next, because these individual changes are relatively small and monotonic, we focus primarily on the differences between the baseline and full models. Looking first at the odds of Asian respondents reporting being dissatisfied, we can see that their decreased risk of dissatisfaction is slightly attenuated as we move from model 1 to model 6 . It appears that when factors such as employment and marital status, health, and income are controlled for-factors on which Asians generally score higher on (see Table 1) and which the literature has shown to be important contributors to SWB - the decreased likelihood of Asian dissatisfaction is slightly reduced but remains substantively and statistically significant.

Turning to the relationship between being Latinx and being dissatisfied, we see a very different pattern. While there are no statistically significant differences in SWB between Latinx and whites before controlling for social and economic characteristics, as soon as we include these covariates in the model Latinx respondents become significantly less likely to report being dissatisfied. In fact, after controlling for employment and marital status, income, health and state 
of residence, Latinx respondents' odds of dissatisfaction are almost half $(\mathrm{OR}=.55)$ that of their white counterparts. This suggests that the lower likelihood of Latinx dissatisfaction is suppressed without considering the effect of known social and economic correlates of SWB, correlates that Latinx respondents generally report lower levels of compared to whites (see Table 1).

Shifting our attention to the likelihood of being "very satisfied" (Section B, Table 3), adding the covariates to the model has relatively little effect on the Asian/white difference. In the baseline model (model 1), Asians have 23\% lower odds whereas in the full model (model 6) their odds are $31 \%$ lower of being "very satisfied". That is, after controlling for employment, marriage, health, income and state of residence, Asian respondents on average were even more less likely than whites to report being "very satisfied" with their lives. In contrast, while Latinx respondents had $22 \%$ lower odds of being "very satisfied" in the baseline model, after controlling for other factors they had 23\% (OR=1.23) higher odds of being "very satisfied" with their lives than their white counterparts. Much of this attenuation and then reversal seems to be the result of income and health. Once these factors, which have been demonstrated to be some of the most important correlates of individual SWB, are controlled for, Latinx respondents demonstrated a significant SWB advantage.

Figure 1. Predicted probabilities of life satisfaction before and after covariate adjustment

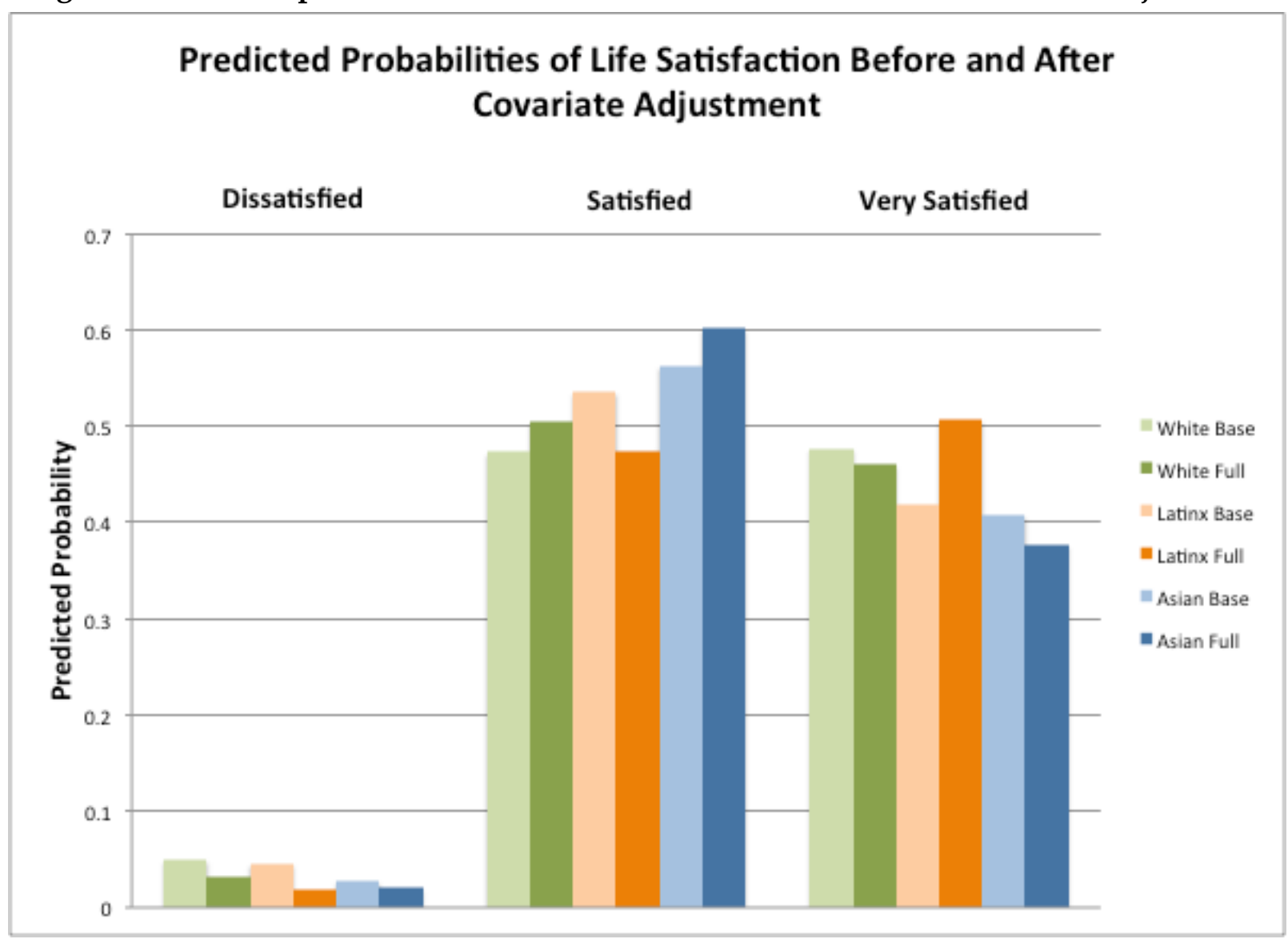

Figure 1 provides a visual representation of these changes based on predicted probabilities before and after adjusting for the covariates. To generate these values, we calculate the probability of each category of the outcome from the baseline (Model 1) and full (Model 6) model coefficients, varying only race/ethnicity. All other variables are set at their means. Note that this strategy may actually under-represent the actual disparities present in the population, on average, between 
racial groups, given that racial and ethnic group means for these covariates are different. However, we believe using the overall covariate means isolates the remaining racial/ethnic differences more clearly and simplifies comparisons for the sake of examining how covariate adjustment increases or reduces disparities in life satisfaction.

Looking first at the "Dissatisfied" columns, we can see that the probability of being dissatisfied goes down for all groups when we control for covariates, but that the decrease is largest for Latinx and smallest for Asian respondents. Turning to the probability of being satisfied, controlling for social and economic characteristics and state of residence increases the probability of being satisfied for whites and Asians but decreases it for Latinx respondents. As changes in these categories are relative (i.e. if probabilities increase in one or two categories they have to decrease in another) we should not be surprised to see a relatively small decrease in the probability of Asians and whites being very satisfied and a relatively large increase in the likelihood of Latinx respondents reporting the highest level of life satisfaction after we control for the covariates.

Our analyses suggest that social and economic characteristics associated with SWB are essential to understanding differences in SWB across racial and ethnic groups, but that controlling for these factors does little to eliminate differences, and in some cases exacerbates them. For instance, controlling for social and economic factors can help explain previous findings that suggested lower levels of SWB among Latinx respondents (Coverdill et al. 2011), but they cannot explain why, after controlling for these factors, Latinx are much less likely to be dissatisfied and much more likely to be very satisfied with their lives - in other words, social and economic characteristics cannot explain why Latinx respondents experience higher overall life satisfaction than their white counterparts. These findings are quite different than what we see among the Asian respondents. While controlling for social and economic factors slightly shifts the relative differences in the probabilities of Asians and whites reporting low and high levels of life satisfaction, it does little to change the general pattern of Asians being much less likely to report being either dissatisfied or very satisfied with life.

One interpretation of our findings is that remaining racial and ethnic differences are the result of other transient characteristics that were not included in the models. However, we would argue that we have included indicators representing most of the major characteristics that have been consistently shown to influence SWB (Dolan et al. 2007). Yet, given the nature of cross-sectional analysis, we cannot rule out this possibility. Another potential explanation is that disparities are due to other, less studied characteristics that differ across race and ethnicity and have not been accounted for in our analyses.

As hypothesized above, we find arguments for the importance of more stable factors related to culture and group experiences much more compelling than those related to genetic or biological factors when it comes to explaining remaining group differences in SWB. Latinx and Asians as a group in the U.S. share a variety of experiences related to the immigrant experience and associated discrimination and prejudice, which have been much less common among the white population in recent history. While scholars have long studied how these obstacles contribute to socioeconomic disadvantage for some racial and ethnic groups, there are likely to be psychological impacts not captured by these types of time-varying social and economic measures. Such impacts may accrue in individuals who experience these things directly, or for larger segments of the population through collective trauma and/or cultural identity. However, we would expect such factors to contribute to lower levels of SWB, in general, despite observing higher levels of SWB among these two minority groups after adjustment for key socioeconomic characteristics (with the exception of Asians' lower likelihood of being very satisfied). While it is 
possible that this is the result of stronger group cohesion in the face of negative group experiences such as prejudice or discrimination, we have no way of empirically examining this assumption.

However, there may be other relatively stable factors stemming from cultures of origin that contribute to differences in life satisfaction. As discussed above, these may be the result of important cultural differences in how people experience and express emotions and/or cultural differences pertaining to the influence of various factors or characteristics. While we are hesitant to offer "culture" as a post-hoc catch-all explanation for differences that have not been explained by more structural variables, the BRFSS does provide us the opportunity to gain some initial leverage on the potential role of culture of origin by including information about the language that was used in the survey interview in our models. Previous research has used similar data as an indicator of assimilation (Alba et al. 2002), where respondents who have greater facility or choose to speak English when given other options are considered more assimilated than respondents who use another language, which is usually assumed to be their native language (Wadsworth and Kubrin 2007). This additional source of measurable, cultural variation provides the potential for more deeply understanding the remaining SWB differences between whites, Latinx and Asians. Based on this assumption, it seems reasonable to suggest that those who were interviewed in their native language are likely to be more deeply embedded in the culture of their, or their family's, place of origin, which may influence emotional norms or experiences expressed through ratings of SWB.

To explore the possibility that cultural assimilation influences SWB, we ran additional models with dummy variables indicating if the interview was done in English, Spanish or a language other than English or Spanish, as well as interactions between Latinx and Spanish language interview, and Asian and non-English/non-Spanish interview. While we do not have information concerning which non-English language other than Spanish was used in an interview, it seems safe to assume that Asian respondents who were interviewed in a non-English language were interviewed in an Asian language. This assumption was supported by higher rates of "other language" interviews among Asians than any other racial/ethnic group. While the rate of nonEnglish interviews among Asians in the sample seems low (3\%) in comparison to population estimates of the language spoken at home among Asians in the US during this time period from the American Community Survey (ACS; about 70\%), it is comparable to the amount (about 3\%) who are classified in the ACS as Limited English Proficient and "do not speak English at all" (Ruggles et al., 2020), which may be the case for participants electing to answer the survey in a language other than English.

Not surprisingly, interacting Latinx with Spanish language interview created significant collinearity when Spanish Language was included in a model with the interaction, as the only respondents interviewed in Spanish were Latinx. For this reason, we dropped the main effect of Spanish Language interview in the second model and let all of the effect of these two variables together be represented by the interaction term, as a measure of stronger adherence to Latinx culture. Table 3 displays the results of these models. Model 1 in Table 3 provides odds ratios and standard errors for the key variables from Model 6 of Table 2, with language of interview added, and Model 2 includes the language and race/ethnicity interactions. While the models include all the covariates of the fully specified model from Table 2, we suppress the output for most covariates to avoid redundancy. Looking at Model 2, only one of the 4 interaction terms is significant. Latinx respondents who were interviewed in Spanish were even less likely than those interviewed in English to report being dissatisfied with their lives. To determine the net impact of these interactions, we calculate a percentage difference between groups by dividing the predicted probability of the outcome for a comparison group (English-speaking Latinx, English- 
Table 3. Ordinal logistic regression models predicting life satisfaction by respondent characteristics and language of interview, BRFSS 2010

\begin{tabular}{|c|c|c|c|c|}
\hline \multirow[b]{3}{*}{ Section A: "Dissatisfied" } & \multicolumn{2}{|l|}{ Model 1} & \multicolumn{2}{|l|}{ Model 2} \\
\hline & Odds Ratio & & Odds Ratio & \\
\hline & (se) & & (se) & \\
\hline \multicolumn{5}{|l|}{ Racel Ethnicity } \\
\hline \multirow[t]{2}{*}{ Asian } & 0.67 & ** & 0.67 & $* *$ \\
\hline & $(0.23)$ & & $(0.23)$ & \\
\hline \multirow[t]{2}{*}{ Latinx } & 0.68 & $* * *$ & 0.68 & $* * *$ \\
\hline & $(0.13)$ & & $(0.13)$ & \\
\hline \multicolumn{5}{|l|}{ Language of Interview } \\
\hline \multirow[t]{2}{*}{ Spanish } & 0.51 & $* * *$ & - & \\
\hline & $(0.29)$ & & - & \\
\hline \multirow[t]{2}{*}{ Other Language } & 1.30 & & 1.56 & \\
\hline & $(0.32)$ & & $(0.45)$ & \\
\hline \multirow[t]{2}{*}{ Latinx * Spanish } & & & 0.52 & $* * *$ \\
\hline & & & $(0.29)$ & \\
\hline \multirow[t]{2}{*}{ Asian * Other Language } & & & 0.82 & \\
\hline & & & $(1.02)$ & \\
\hline \multicolumn{5}{|l|}{ Section B: "Very Satisfied" } \\
\hline \multicolumn{5}{|l|}{ Racel Ethnicity } \\
\hline \multirow[t]{2}{*}{ Asian } & 0.70 & $* * *$ & 0.70 & $* * *$ \\
\hline & $(0.04)$ & & $(0.04)$ & \\
\hline \multirow[t]{2}{*}{ Latinx } & 1.20 & $* * *$ & 1.20 & $* * *$ \\
\hline & $(0.04)$ & & $(0.05)$ & \\
\hline \multicolumn{5}{|l|}{ Language of Interview } \\
\hline \multirow[t]{2}{*}{ Spanish } & 1.03 & & - & \\
\hline & $(0.06)$ & & - & \\
\hline \multirow[t]{2}{*}{ Other Language } & 0.42 & * & 0.16 & $* * *$ \\
\hline & $(0.17)$ & & $(0.08)$ & \\
\hline \multirow[t]{2}{*}{ Latinx * Spanish } & & & 1.02 & \\
\hline & & & $(0.06)$ & \\
\hline \multirow[t]{2}{*}{ Asian * Other Language } & & & 2.89 & \\
\hline & & & $(1.95)$ & \\
\hline Cut 1 Intercept & 0.02 & & 0.02 & \\
\hline Cut 2 Intercept & 0.50 & & 0.50 & \\
\hline $\mathrm{N}$ & 321417 & & 321417 & \\
\hline
\end{tabular}

Notes: Models adjust for sampling weights and state fixed effects

* $\mathrm{p}<0.05$

** $\mathrm{p}<0.01$

$* * * p<0.001$

speaking whites) over the same probability for Spanish-speaking Latinx and subtract the result from 1. Holding all else constant, Spanish-speaking respondents were $48 \%$ ( $1-(.012 / .023))$ and 
$30 \%(1-(.023 / .033))$ less likely to report being dissatisfied, relative to their English speaking Latinx and white counterparts, respectively. Given the inconsistency of this finding across categories of the outcome and language/ethnic group combinations, we encourage a cautious interpretation. However, it does suggest that the higher average life satisfaction of Latinx respondents may be at least partly associated with more cultural, rather than socio-economic, characteristics. Does the fact that the interaction representing Asian respondents being interviewed in another language is not significant suggest a less relevant role of culture for Asians in terms of SWB? We are hesitant to move towards this conclusion, in part because the percentage of Asian respondents interviewed in a non-English language was only three percent, whereas forty-four percent of Latinx respondents were interviewed in Spanish, resulting in very limited power to detect a meaningful association.

However, another possible explanation for both significant and non-significant findings is that English language proficiency may actually capture different nationalities of origin rather than different levels of assimilation. Both the terms "Latinx" and "Asian" represent very heterogeneous populations from various countries of origin that may have higher or lower average levels of life satisfaction, and which face very different constraints in the U.S. For example, if more recent Latinx immigrants who are less likely to speak English are from countries that tend to have higher average SWB, findings may reflect cultural differences in country of origin rather than assimilation. Likewise, among Asian groups, findings may be muddled by tremendous variability in country of origin. The average experiences of recent Hmong and Japanese immigrants in the U.S., for example, are potentially very different despite a perhaps similar likelihood of speaking a non-English language during their interview. It is unfortunately impossible with the present data, however, to parse out these compositional issues that could explain the preliminary findings around language and SWB.

\section{Discussion and conclusion}

Our findings suggest that common socioeconomic and demographic characteristics are important in understanding racial and ethnic differences in life-satisfaction, but that these factors alone are insufficient for explaining the differences between white, Asian, and Latinx respondents. There are clearly other, potentially more stable factors, that drive some of the racial and ethnic differences in SWB. We offer preliminary evidence that some of these factors stem from cultural differences, like language, but face data limitations for empirically identifying exactly what these cultural factors are.

In considering Latinx/white differences in life satisfaction, we come to a somewhat different conclusion than was reached by Coverdill et al. (2011) in their study of happiness among GSS respondents. Some, but not all, of the ethnic differences in SWB can be explained by other social and economic characteristics of the respondents. More specifically, factors such as marriage, income, education, employment and health can explain why zero-order correlations suggest a life-satisfaction advantage (in terms of the likelihood of being very satisfied) of white over Latinx respondents, but these same factors cannot explain why, after controlling for them, we see a significant SWB advantage of Latinx respondents over whites. This advantage is present at both the low (with a lower likelihood of being dissatisfied) and the high (with a higher likelihood of being very satisfied, compared to whites) end of the life satisfaction spectrum. Based on our preliminary findings concerning the role of language, we suggest that some of this Latinx advantage likely stems from more cultural factors. Just as Latinx respondents demonstrated a lower likelihood than whites of being dissatisfied, Spanish-speaking Latinx respondents reported less dissatisfaction than their English-speaking Latinx counterparts. While these 
findings are preliminary and must be interpreted with caution, they are consistent with a wealth of research focusing on the "Hispanic health paradox" which finds higher than expected levels of a variety of health and well-being indicators among Latinx groups, given their otherwise lower rankings on socio-economic indicators typically associated with positive outcomes (Sorlie et al. 1993; Cervantes 1996; Wadsworth and Kubrin 2007). This literature has often pointed to a stronger sense of family, community, religion, and group identity as the cultural factors driving the paradox in health and well-being outcomes. These same factors could certainly be the foundation of higher levels of life-satisfaction as well, though they are not measured directly in the BRFSS data. The findings are also consistent with recent work on SWB in Latin American (Rojas and Vega 2017; Beytia 2016) in suggesting that cultural factors boost happiness and life satisfaction among respondents who appear to be disadvantaged by other correlates of SWB. Investigating these differences more thoroughly, both cross-nationally and within U.S. samples would be a fruitful avenue for future research.

For Asians, we provide an initial examination of SWB differences using the largest sample to date and identify patterns that were quite different than those typically observed among blacks and Latinx. While very different than the comparison between white and Latinx respondents, the findings pertaining to Asians also hint that more cultural aspects may play an important role in shaping the SWB of Asians. While there were relatively small changes in the Asian/white differences in reporting both high and low levels of life-satisfaction as we controlled for more socio-economic factors, we think the most intriguing finding was how robust the differences were to the addition of theoretically important covariates of life-satisfaction. That differences were seen on both ends of the spectrum, with Asians being significantly less likely to report being very satisfied or dissatisfied, reveals a modal, more moderate level of satisfaction amongst this group than we observe in other racial or ethnic groups. While our additional analyses that added indicators of language to the models did not offer additional information regarding this pattern among Asian respondents, it does seem consistent with previous research that has found broad differences in SWB between respondents in western vs. eastern countries (Diener and Suh 1999; Diener and Oishi, 2000). This previous work has pointed towards different cultural norms regarding emotional expression (Tov and Diener 2009). While this previous research focused more on demonstrating the lower likelihood of respondents from Asian countries viewing high SWB expression as important, it may simply be that a certain middle ground is viewed as more desirable than either especially high or low SWB. This same process could be playing out among Asian respondents living in the U.S.

We view our analyses as providing a starting point for examining the importance of more cultural factors. Even after controlling for social and economic characteristics that are strongly associated with levels of assimilation, our analyses suggest that being Latinx, and even more so, speaking Spanish, is associated with higher life satisfaction. While language is not an ideal proxy for assimilation, we view these findings as preliminary evidence that more culturally based, traitlike factors are essential for considering the SWB of racial and ethnic minorities. That the patterns of association regarding Latinx respondents are consistent with existing research on the Hispanic Health Paradox and SWB in Latin America is affirming. A deeper understanding of racial and ethnic differences in SWB may be possible with further empirical investigation of other cultural factors, such as stronger emphasis on family and community, group identification and/or other culturally-based values, which may be present in other datasets.

Our findings regarding Asians also speak to the importance of culture, though we have less empirical evidence that differences in SWB are related to assimilation (or lack thereof). Instead, our findings are similar to those of other cross-national work that has pointed to differences in 
cultural expression norms. Whether these differences are manifestations of divergent psychological experiences, or simply a reflection of how individuals interpret or relate their experiences cannot be addressed with the current data. Better understanding the source of these remaining disparities is an important avenue for future research.

Collectively, our findings add to, and extend, the research on SWB among racial and ethnic minorities in the U.S. That some of the baseline differences in SWB between Latinx, Asian, and white respondents can be explained by inequalities on a variety of social and economic determinants offers further evidence to the immense body of work regarding the impacts of racial/ethnic stratification. That other differences cannot be explained by these determinants speaks to the importance of culture, a topic that has received less attention in the literature on SWB. Future research should further investigate these types of factors if we hope to better understand what makes people happier and more satisfied with their lives.

\section{Acknowledgments}

BRFSS Data were made available by the Centers for Disease Control and Prevention.

\section{Conflict of interest statement}

The authors report no conflicts of interest.

\section{Authors' Contributions}

Both authors made critical contributions to all aspects of the project.

\section{Authors}

Tim Wadsworth

University of Colorado Boulder

tim.wadsworth@colorado.edu

Philip M. Pendergast

U.S. Census Bureau

\section{Disclaimer}

Any opinions and conclusions expressed herein are those of the author and do not necessarily reflect the views of the U.S. Census Bureau.

\section{Publishing Timeline}

Received 14 October 2020

Revised version received 10 February 2021

Accepted 16 February 2021

Published 31 May 2021

\section{References}

Alba, R., Logan, J., Lutz, A., \& Stults, B. (2002). Only English by the third generation? Loss and preservation of the mother tongue among the grandchildren of contemporary immigrants. Demography, 39(3), 467-484. https://doi.org/10.1353/dem.2002.0023

Alexander, J. C., Eyerman, R., Giesen, B., Smelser, N. J., \& Sztompka, P. (2004). Cultural Trauma and Collective Identity. Berkeley, Calif: University of California Press. https://doi.org/10.1525/9780520936768

Andrews, F. M., \& Withey, S. B. (1976). Social Indicators of Well-Being. Americans' Perceptions of Life Quality. New York: Plenum Press. https://doi.org/10.1007/978-1-4684-2253-5 
Barger, S. D., Donoho, C. J., \& Wayment, H. A. (2009). The relative contributions of race/ethnicity, socioeconomic status, health, and social relationships to life satisfaction in the United States. Quality of Life Research, 18(2), 179-189. https://doi.org/10.1007/s11136-008-9426-2

Bartels, M. (2015). Genetics of wellbeing and its components satisfaction with life, happiness, and quality of life: A review and meta-analysis of heritability studies. Behavioral Genetics, 45(2), 137-156. https://doi.org/10.1007/s10519-015-9713-y

Beytia, P. (2016). The Singularity of Latin American Patterns of Happiness. In: Rojas, M. (ed.) Handbook of Happiness Research in Latin America. Springer, Dordecht. https://doi.org/10.1007/978-94-017-7203-7

Blanchflower, D. G., \& Oswald, A. J. (2004). Well-being over time in Britain and the USA. Journal of Public Economics, 88(7-8), 1359-1386. https://doi.org/10.1016/s0047-2727(02)00168-8

Bracy, J. (1976). The quality of life experience of Black people. In A. Campbell, P. E. Converse, \& W. L. Rodgers (Eds.), The Quality of American Life: Perceptions, Evaluations, and Satisfactions. New York: Russell Sage Foundation. https://doi.org/10.1093/sf/56.1.283

Campbell, A., Converse, P. E., \& Rodgers, W. L. (1976). The Quality of American Life: Perceptions, Evaluations, and Satisfactions. Russell Sage Foundation. https://doi.org/10.7758/9781610441032

Centers for Disease Control and Prevention (CDC). Behavioral Risk Factor Surveillance System Data. Atlanta, Georgia: U.S. Department of Health and Human Services, Centers for Disease Control and Prevention, 2005-2008. https://doi.org/10.3886/icpsr34085

Cervantes, A. (1996). Latinos in the United States and Chicago: The history, demographics, and prospects of a growing population. The Chicago Project News 2(3), 1-4.

Clark, A. E., Frijters, P., \& Shields, M. A. (2008). Relative income, happiness, and utility: An

explanation for the Easterlin Paradox and other puzzles. Journal of Economic Literature, 46(1), 95-144. https://doi.org/10.1257/jel.46.1.95

Clemente, F., \& Sauer, W. J. (1976). Life satisfaction in the United States. Social Forces, 54(3), 621-631. https://doi.org/10.1093/sf/54.3.621

Coverdill, J. E., Lopez, C. A., \& Petrie, M. A. (2011). Race, ethnicity and the quality of life in America, 1972-2008. Social Forces, 89(3), 783-805. https://doi.org/10.1353/sof.2011.0002

Davis, J. A., Smith, T. W., \& Marsden, P. V. (2009). General Social Surveys, 1972-2008. [machine-readable data file]. Chicago, IN: National Opinion Reach Center. https://doi.org/10.3886/ICPSR36797.v1

Diener, E., \& Oishi, S. (2000). Money and happiness: Income and subjective well-being across nations. In E. Diener \& E. M. Suh (Eds.), Culture and subjective well-being (pp. 185-218). Boston, MA: MIT Press. https://doi.org/10.7551/mitpress/2242.001.0001

Diener, E., \& Ryan, K. (2009). Subjective well-being: A general overview. South African Journal of Psychology, 39(4), 391-406. https://doi.org/10.1177/008124630903900402

Dolan, P., Peasgood, T., \& White, M. (2008). Do we really know what makes us happy? A review of the economic literature on the factors associated with subjective well-being. Journal of Economic Psychology, 29(1), 94-122. https://doi.org/10.1016/j.joep.2007.09.001

Diener, E \& Suh, E. M. (1999). National differences in subjective well-being (Vol. Well-being: The foundations of hedonic psychology). New York: Russell Sage Foundation. https://doi.org/10.1017/s0953820806231972

Eid, M., \& Diener, E. (2001). Norms for experiencing emotions in different cultures: Inter- and intranational differences. Journal of Personality and Social Psychology, 81(5), 869-885. https://doi.org/10.1037/0022-3514.81.5.869

Ferrer-i-Carbonell, A., \& Frijters, P. (2004). How important is methodology for the estimates of the determinants of happiness? The Economic Journal, 114(497), 641-659. https://doi.org/10.1111/j.14680297.2004.00235.x

Graham, C., \& Pinto, S. (2019). Unequal hopes and lives in the USA: Optimism, race,

place, and premature mortality. Journal of Population Economics, 32, 665-733.

https://doi.org/10.1007/s00148-018-0687-y

Haring-Hidore, M., Stock, W. A., Okun, M. A., \& Witter, R. A. (1985). Marital status and subjective wellbeing: A research synthesis. Journal of Marriage and the Family, 47(4), 947.

https://doi.org/10.2307/352338 
Herzog, A. R., Rodgers, W. L., \& Woodworth, J. (1982). Subjective Well-Being among Different Age Groups. Ann Arbor: University of Michigan.

Hughes, M., \& Thomas, M. E. (1998). The continuing significance of race revisited: A study of race, class, and quality of life in America, 1972 to 1996. American Sociological Review, 63(6), 785-795. https://doi.org/10.2307/2657501

Iceland, J., \& Ludwig-Dehm, S. (2019). Black-white differences in happiness, 1972-2014. Social Science Research, 77, 16-29. https://doi.org/10.1016/j.ssresearch.2018.10.004

Karlson, K. B., Holm, A., \& Breen, R. (2012). Comparing regression coefficients between same-sample nested models ssing logit and probit: A new method. Sociological Methodology, 42(1), 286-313. https://doi.org/10.1177/0081175012444861

Kubrin, C. E., Wadsworth, T. P., \& DiPietro, S. (2006). Deindustrialization, disadvantage and suicide among young black males. Social Forces, 84(3), 1559-1579. https://doi.org/10.1353/sof.2006.0052

Mood, C. (2010). Logistic regression: Why we cannot do what we think we can do, and what we can do about it. European Sociological Review, 26(1), 67-82. https://doi.org/10.1093/esr/jcp006

Oswald, A. J., \& Wu, S. (2010). Objective Confirmation of Subjective Measures of Human Well-Being: Evidence from the U.S.A. Science, 327(5965), 576-579. https://doi.org/10.1126/science.1180606

Peterson, C., Park, N., \& Seligman, M. E. P. (2005). Orientations to happiness and life satisfaction: The full life versus the empty life. Journal of Happiness Studies, 6(1), 25-41. https://doi.org/10.1007/s10902-0041278-Z

Rojas, M., García Vega J. J. (2017) Well-Being in Latin America. In: Estes R., Sirgy M. (eds) The Pursuit of Human Well-Being. International Handbooks of Quality-of-Life. Springer, Cham. https://doiorg.colorado.idm.oclc.org/10.1007/978-3-319-39101-4_8

Ruggles, S., Flood, S., Goeken, R., Grover, J., Meyer, E., Pacas, J., \& Sobek, M. (2020). IPUMS USA: Version 10.0 [dataset]. Minneapolis, MN: IPUMS. https://doi.org/10.18128/D010.V10.0

Scollon, C. N., Diener, E., Oishi, S., \& Biswas-Diener, R. (2004). Emotions across cultures and methods. Journal of Cross-Cultural Psychology, 35(3), 304-326. https://doi.org/10.1177/0022022104264124

Sorlie, P. D., Blacklund, M. S., Johnson, N. J., \& Rogot, E. (1993). Mortality by Hispanic status in the United States. Journal of American Medical Association, 20, 2464-2468.

https://doi.org/10.1001/jama.1993.03510200070034

StataCorp. (2013). Stata Statistical Software: Release 13. College Station, TX: StataCorp LP. https://doi.org/10.7717/peerj.4598/supp-10

Stevenson, B., \& Wolfers, J. (2012). Subjective and objective indicators of racial progress. The Journal of Legal Studies, 41(2), 459-493. https://doi.org/10.1086/669963

Thomas, M. E., \& Hughes, M. (1986). The continuing significance of race: A study of race, class, and quality of life in America, 1972-1985. American Sociological Review, 51(6), 830. https://doi.org/10.2307/2095370

Tov, W., \& Diener, E. (2009). Culture and subjective well-being. In P. E. Diener (Ed.), Culture and WellBeing (pp. 9-41). Springer Netherlands. https://doi.org/10.1007/978-90-481-2352-0_2

Wadsworth, T. (2016). Marriage and subjective well-being: How and why context matters. Social Indicators Research, 126(3), 1025-1048. https://doi.org/10.1007/s11205-015-0930-9

Wadsworth, T., \& Kubrin, C. E. (2007). Hispanic suicide in U.S. metropolitan areas: Examining the effects of immigration, assimilation, affluence, and disadvantage. American Journal of Sociology, 112(6), 18481885. https://doi.org/10.1086/512711

Wadsworth, T., \& Pendergast, P. M. (2014). Obesity (sometimes) matters: The importance of context in the relationship between obesity and life satisfaction. Journal of Health and Social Behavior, 55(2), 196214. https://doi.org/10.1177/0022146514533347

Williams, D. R., \& Sternthal, M. (2010). Understanding racial/ethnic disparities in health: Sociological contributions. Journal of Health and Social Behavior, 51(Suppl), S15-S27.

https://doi.org/10.1177/0022146510383838

Williams, R. (2016). Understanding and interpreting generalized ordered logit models. The Journal of Mathematical Sociology, 40(1), 7-20. https://doi.org/10.1177/0022146510383838 
Wilson, W. J. (1990). The Truly Disadvantaged: The Inner City, the Underclass, and Public Policy (Reprint edition). Chicago: University of Chicago Press.

https://doi.org/10.7208/chicago/9780226924656.001.0001

Wilson, W. J. (1980). The Declining Significance of Race: Blacks and Changing American Institutions. Chicago; London: University of Chicago Press. https://doi.org/10.1093/sf/57.3.991 